\title{
Near infrared low-resolution spectra of 7 Be stars and AG Carinae ${ }^{\star, \star \star}$
}

\author{
R.E. Mennickent ${ }^{1}$ and C. Sterken ${ }^{2, \star \star \star}$ \\ 1 Facultad de Ciencias Físicas y Matemáticas, Departamento de Física, Casilla 4009, Concepción, Chile \\ rmennick@gauss.cfm.udec.cl \\ ${ }^{2}$ University of Brussels (VUB), Pleinlaan 2, 1050 Brussels, Belgium \\ csterken@vub.ac.be
}

Received April 9; accepted May 22, 1996

\begin{abstract}
We present and discuss $\lambda 6470-8780$ spectra of 7 poorly-studied Be stars and of one Luminous Blue Variable (AG Car), and give equivalent widths and radial velocities of selected spectral lines.
\end{abstract}

Key words: stars: individual — stars: variables — stars: oscillations - stars: Be

\section{Introduction}

In this data paper we present near-infrared $(\lambda 6470-$ -8780) spectra of 7 poorly-studied Be stars and of one Luminous Blue Variable (AG Car) together with equivalent widths and radial velocities of the spectral lines. These data will be useful for statistical purposes, long-term studies and also for selecting specific targets for high-resolution observations.

Previous surveys of Be stars have shown the presence of several lines of astrophysical interest in the near-infrared spectral region: neutral hydrogen $(\mathrm{H} \alpha$ and Paschen lines), neutral helium $(\lambda 6678,7281)$, neutral oxygen $(\lambda 7772-7774-7775$ and $\lambda$ 8446) and ionized calcium $(\lambda 8542-8662-8498 \AA)$. NI $\lambda 8686-8683-8680$ and $\lambda 8719-8712-8703$ and FeII $\lambda 7712$ have been observed occasionally (see, for example, Andrillat et al. 1988).

Send offprint requests to: R.E. Mennickent

* Based on observations obtained at Las Campanas Observatory and at the European Southern Observatory in Chile

** Table 2 is only available in electronic form at the CDS via anonymous ftp 130.79.128.5 or via WWW at http://cdsweb.ustrasbg.fr/Abstract.html

$\star \star \star$ Belgian Fund for Scientific Research (NFWO)

\section{Observations and measurements}

The spectra were obtained with the $2.5 \mathrm{~m}$ Du Pont telescope at Las Campanas Observatory (Chile) on May 20 and 21, 1991 (UT date). This observing time was allocated for studying faint cataclysmic variable stars, but bad weather forced us to carry out the backup program consisting of observations of bright Be stars. The stars, selected from the Supplement to the Bright Star Catalogue (Hoffleit et al. 1983), are listed in Table 1. We used the CRAF CCD array of $1024 \times 102412 \mu$ pixels, characterized by a gain of $1.24 \mathrm{e}^{-} / \mathrm{ADU}$ and a readout noise of $7.7 \mathrm{e}^{-}$. A grating of $600 \mathrm{l} / \mathrm{mm}$ tilted at an angle of 29.65 yielded a projected spectral range of $6470-8780 \AA$. A slit width of 0.77 was selected, giving a spectral resolution of $5.4 \AA$. The CCD data were reduced with IRAF standard routines involving bias subtraction, flat field correction and subtraction of one-dimensional spectra after removing the night sky. He-Ne-Ar exposures were taken after each object spectrum giving wavelength calibrations with a typical rms scatter of $0.02 \AA$. Non-photometric weather conditions prevented us of obtaining flux calibrated spectra. Normalized spectra were obtained by fitting the continuum with a high order polynomial, the resulting data are given in Table 2. Equivalent widths (W) were measured between the points where the line flux was equal to the continuum, using the "e" routine in the IRAF "splot" package. Internal errors were of the order of $2 \%$. Due to the frequent passage of cirrus, it was difficult to optimally tune the exposure time for HD 124834, HD 179419 and HD 203699, a situation that led to some top-saturated $\mathrm{H} \alpha$ profiles. For these stars a lower limit for $W_{\alpha}$ was then obtained. The results are given in Table 3 . In the case of emission profiles superimposed on absorption wings, equivalent widths were also measured upon the points defining the minima of the emission lines. These measurements are indicated in Table 3 with a preceding slash. In addition, radial velocities were calculated by fitting the 
profiles with a gaussian function. Formal errors, derived from the rms scatter of the wavelength calibrations, are of the order of $0.3 \mathrm{~km} \mathrm{~s}^{-1}$ while internal errors due to the method of measurement are of the order of $1 \mathrm{~km} \mathrm{~s}^{-1}$. The radial-velocity results are listed in Table 4 and they refer to the Local Standard of Rest $\left(v_{\odot}=20 \mathrm{~km} \mathrm{~s}^{-1}, \alpha_{\odot}=18^{\mathrm{h}}\right.$ and $\delta_{\odot}=+30^{\circ}$ ).

The stellar radial velocities of Be stars are commonly derived from the optical helium lines which are not so contaminated by emission. Due to the lack of such lines in our spectral region, it was not possible to correct our measurements for the stellar proper motion. In addition, because the OI $\lambda 7773$ triplet was not resolved in our spectra, we did not measure radial velocities for this line. Spectra of HD 90966, HD 91188 and AG Car are shown in Figs. 1, 2 and 3 for illustration.

Figure 4 gives the position of the $7 \mathrm{Be}$ stars in the Geneva (reddening-free) $X, Y$ space, which is the Geneva observational H-R diagram for hot stars (Cramer \& Maeder 1979) - the solid lines indicate the locus of the early-type main-sequence stars, giants and (Iab) supergiants, and are taken from Cramer (1994).

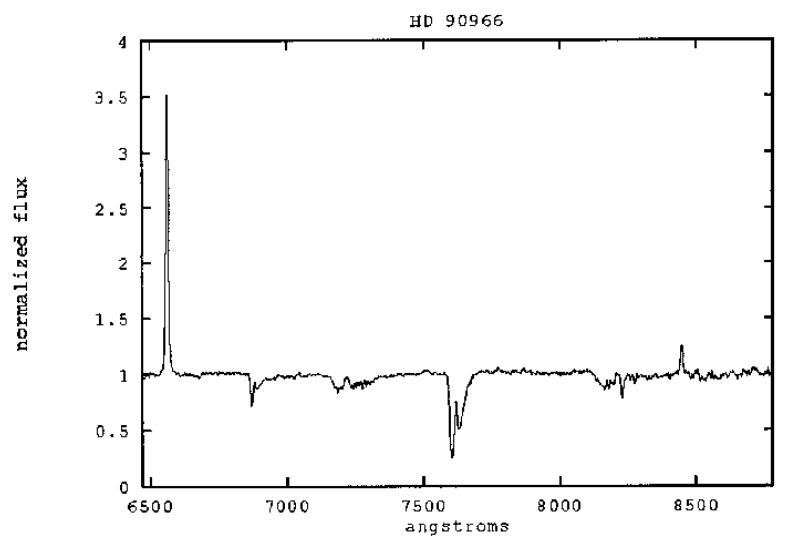

Fig. 1. Normalized spectrum of HD 90966. Note the strong $\mathrm{H} \alpha$ emission and the weaker OI emission at $\lambda 7775$

\section{Results for individual stars}

Our spectra do not cover the region around $\mathrm{H} \beta$, but some information on the strength and variability of the $\mathrm{H} \beta$ line can be derived from published $u v b y \beta$ and Geneva 7-color photometry. We, therefore, have added to Table 1 the observed $\beta$ index, and we calculated the corresponding index $\beta_{\text {calc }}=2.5909+0.0667 X-0.6801 Y-0.2559 X Y+$ $0.1748 X^{2}-2.4676 Y^{2}+0.1448 X Y^{2}+0.2582 X^{2} Y-$ $0.0612 X^{3}+0.4418 Y^{3}$. According to Cramer $(1984,1994)$, the excess $\beta-\beta_{\text {calc }}$ is the signature of the presence of an emission feature in the $\mathrm{H} \beta$ line (a strong and variable excess points to the presence of a strong, respectively

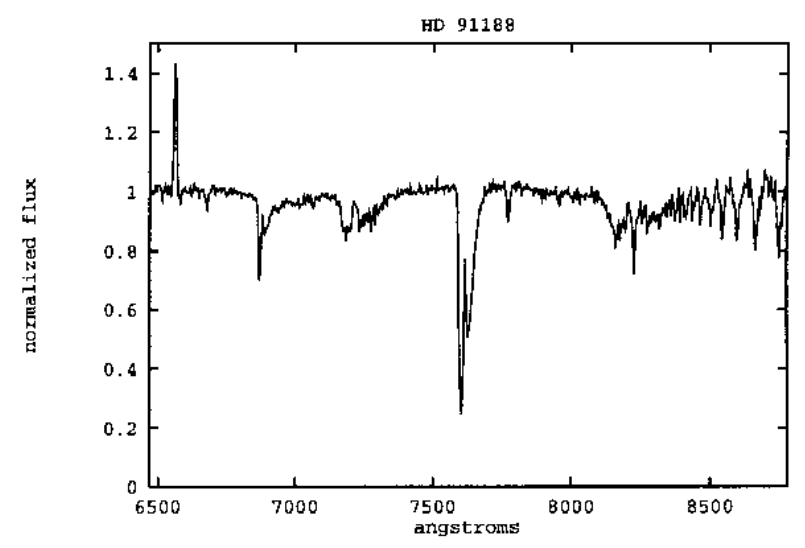

Fig. 2. Normalized spectrum of HD 91188. A weak $\mathrm{H} \alpha$ emission and absorptions at HeI, OI and Paschen lines characterize this spectrum

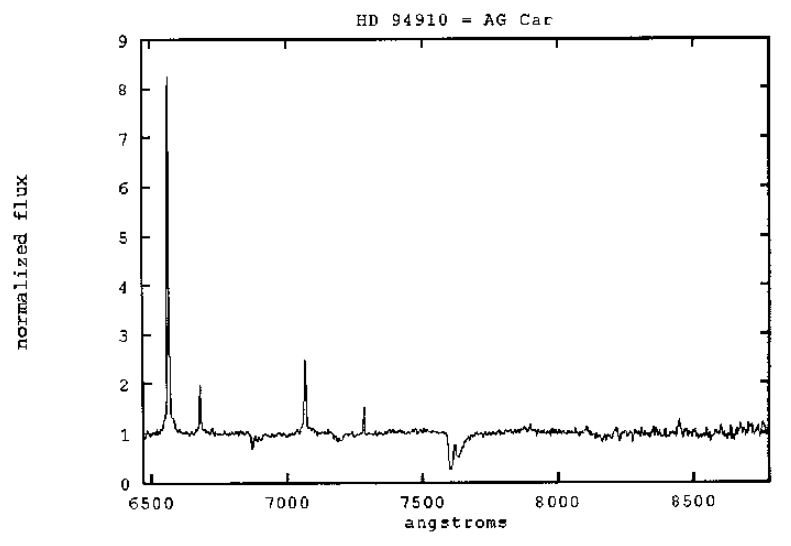

Fig. 3. Normalized spectrum of AG Car. A strong $\mathrm{H} \alpha$ emission peak with broad emission wings dominate the spectrum. Prominent He I emission lines are also visible

variable, emission component). We refer to Fig. 5 for an overview of all our observed $\mathrm{H} \alpha$ line profiles.

\section{1. $H D 90966$}

Emission at $\mathrm{H} \alpha$ and $\mathrm{H} \beta$ during 1957-1963 was reported by Buscombe (1970). Infrared excess was detected by IRAS (Oudmaijer et al. 1992). Despite its rather large $\beta-\beta_{\text {calc }}$, our spectrum shows moderate $\mathrm{H} \alpha$ and OI emissions. He I $\lambda 6678$ is in absorption and Paschen lines seem to be completely filled in by emission.

\section{2. $H D 91188$}

HD $91188=$ SX Vel is a short-period $(P=2$ d 924$)$ photometric variable that was extensively observed by Balona et al. (1992), for a phase diagram see Fig. 6. Years ago Albers (1969) reported emission at $\mathrm{H} \alpha$ and probable emission at OI $\lambda$ 7775. We have found a weak $\mathrm{H} \alpha$ emission while OI $\lambda 7775$ is in absorption. The equivalent width of this line 


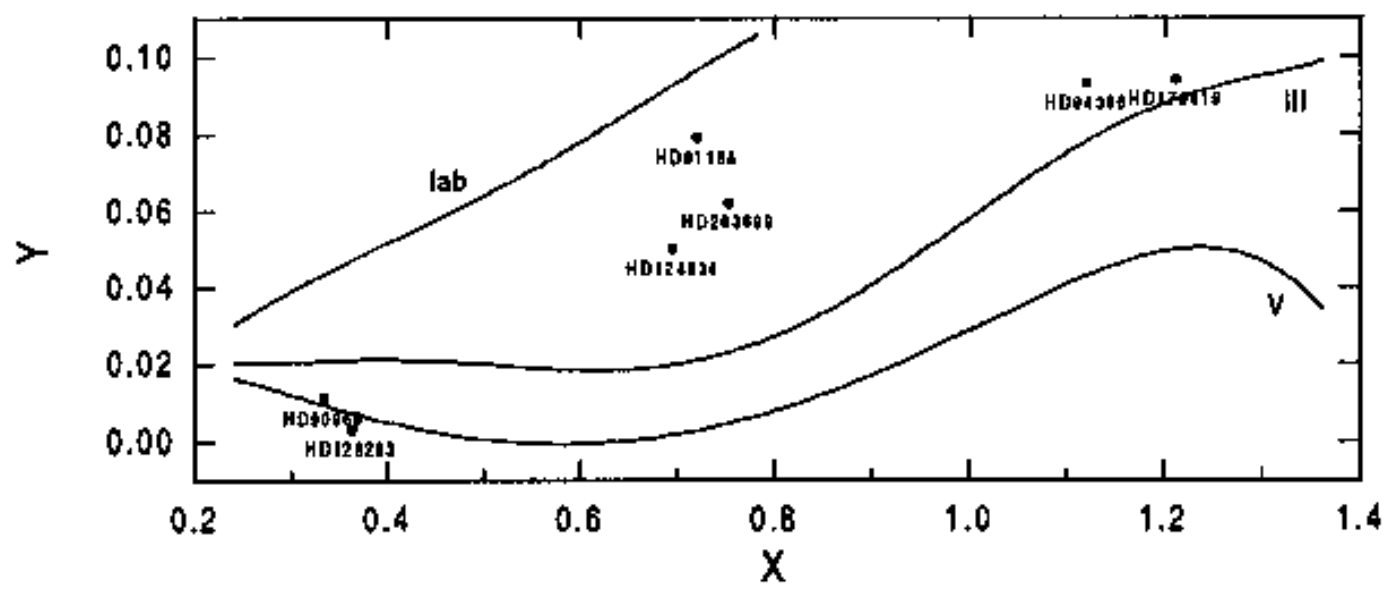

Fig. 4. Position of the 7 Be stars in the Geneva $X, Y$ photometric parameter space, which is the Geneva observational H-R diagram for hot stars (Cramer \& Maeder 1979) — the solid lines indicate the locus of the early-type main-sequence stars, giants and (Iab) supergiants, and are taken from Cramer (1994)
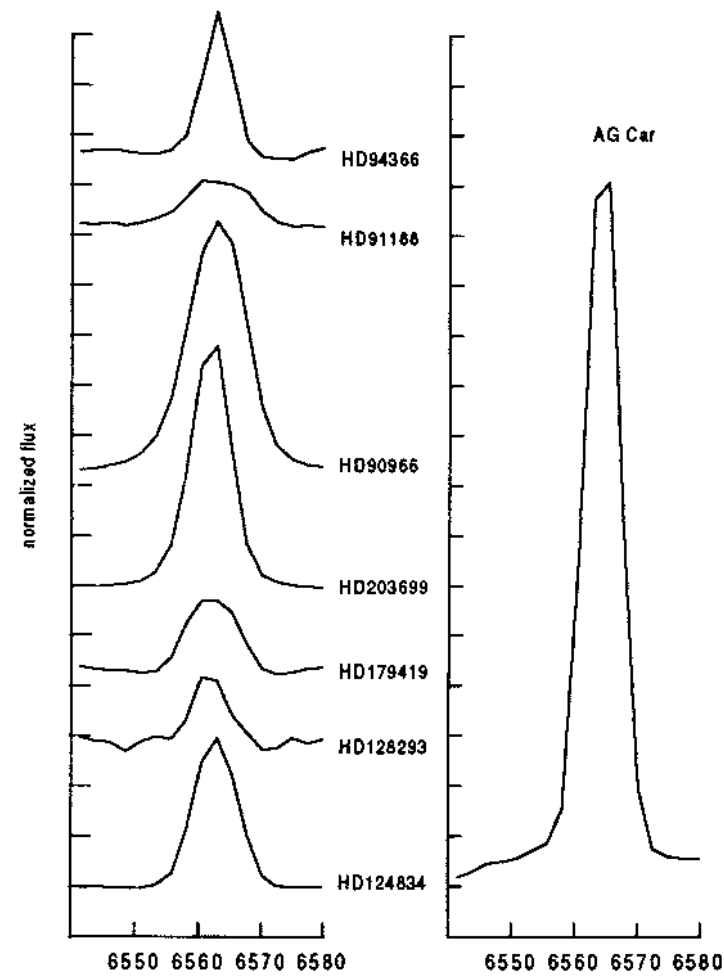

Fig. 5. $\mathrm{H} \alpha$ emission line profiles for $\mathrm{AG}$ Car (right) and for the 7 Be stars on a same intensity scale (tick marks 0.5 apart)

is rather large when compared to the average observed in non-shell Be stars of the same spectral type (Jashek et al. 1993). This could be evidence of a possible transition into a shell phase. He I $\lambda 6678$ and Paschen lines are observed in absorption. In addition, weak $\left(W_{\lambda} \lesssim 0.5 \AA\right) \mathrm{FeI}$ emission at $\lambda 7955$ and 7959 seems to be present. The radial velocities of the P11-P15 Paschen lines show a mean of $19 \pm$

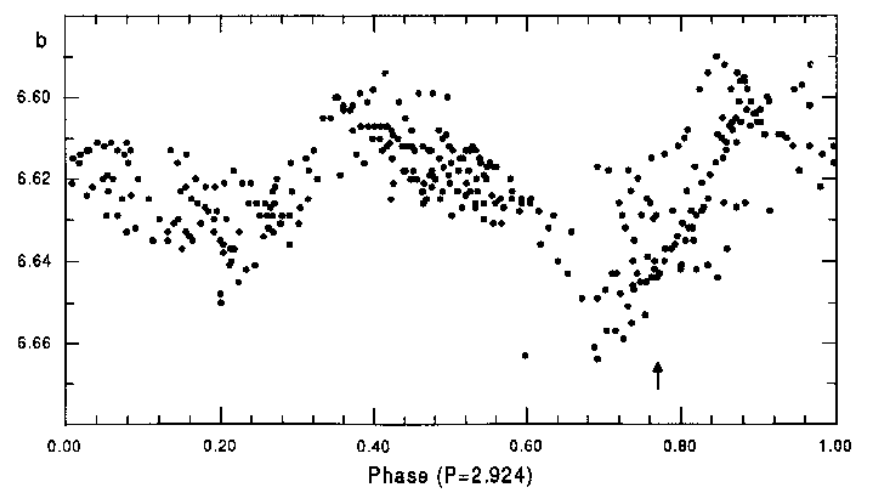

Fig. 6. Phase diagram of Strömgren $b$ data from Balona et al. (1992). The arrow indicates the phase at which our spectrogram was taken. Note the double wave

$11 \mathrm{~km} \mathrm{~s}^{-1}$, slightly larger than the radial velocity derived from the $\mathrm{H} \alpha$ profile $\left(-16 \mathrm{~km} \mathrm{~s}^{-1}\right)$. As absorption lines are formed close to the stellar photosphere and emission lines arise from the circumstellar gas, this fact probably indicates radial gas motions in the envelope.

\section{3. $H D 94366$}

This B6IIIe star shows weak emission at $\mathrm{H} \alpha$ and Paschen lines in absorption. This is a very ill-studied star: the SIMBAD database does not give a single reference to previous studies.

\subsection{HD 94910 (AG Car)}

This is a well-studied Luminous Blue Variable (LBV) surrounded by a prominent ring nebula - see Humphreys \& Davidson (1994) for a review of LBVs including AG Car, 


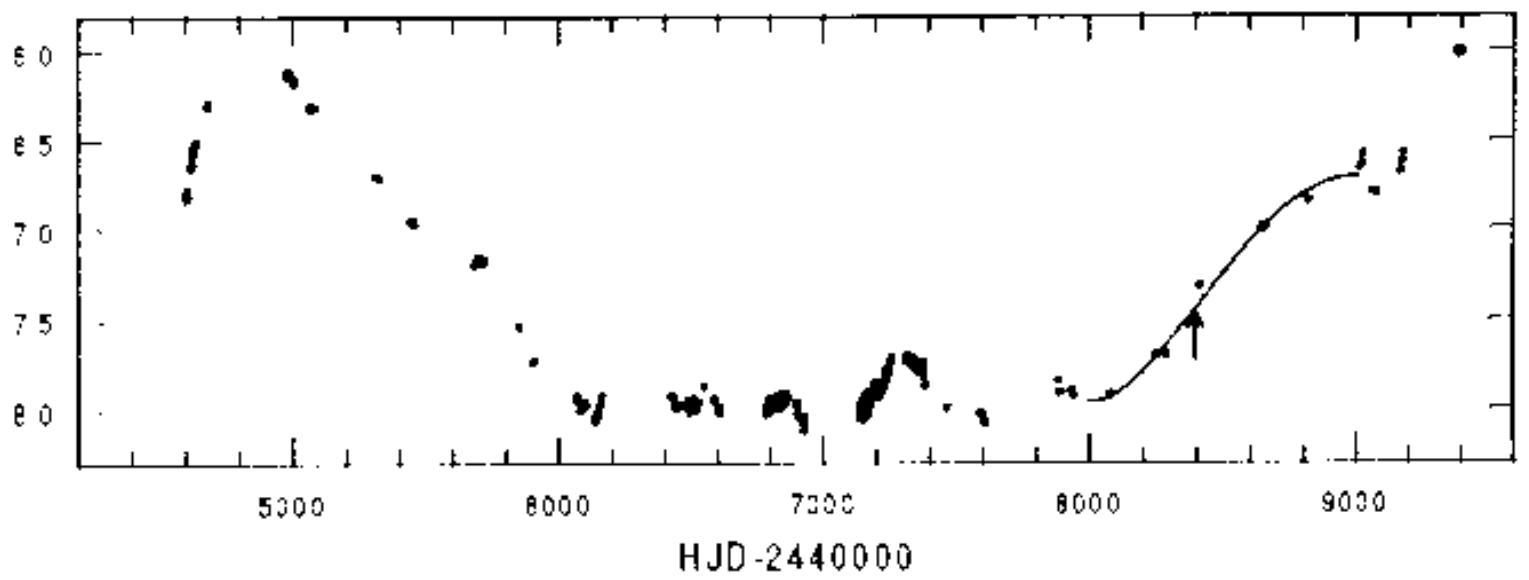

Fig. 7. Composite $V$ light curve of AG Car based on data from miscellaneous sources. The vertical arrow denotes the moment of our observation, the thin line is a hand-drawn curve to illustrate the approximate magnitude level of the star at that epoch

Table 1. The observed stars. The exposure time and the mean signal to noise ratio at the continuum level are given for each spectrum. Magnitudes and spectral types are from Hoffleit et al. (1983). $\beta_{\text {calc }}$ is the calculated $\beta$ index derived from the data published by Rufener (1988), $\beta$ is the observed Strömgren $\beta$ index taken from the catalogue of Hauck \& Mermillod (1990), except for HD 128293 for which the value by Reed (1996) was used

\begin{tabular}{|r|l|l|c|r|r|r|c|}
\hline \multicolumn{1}{c}{ HD } & \multicolumn{1}{c}{$V$} & MK & HJD -24440000 & Exp.(s) & \multicolumn{2}{c}{$S / N$} & \multicolumn{2}{c}{$\beta_{\text {calc }}$} & $\beta$ \\
\hline 90966 & 6.45 & B2/3III:ne & 8396.57533 & 3 & 60 & 2.622 & 2.524 \\
91188 & 6.64 & B3IIIe & 8396.58039 & 10 & 90 & 2.634 & 2.582 \\
94366 & 6.77 & B6IIIe & 8396.58478 & 2 & 40 & 2.719 & \\
94910 & 6.96 & B2:pe & 8396.58777 & 5 & 30 & & \\
124834 & 6.60 & B3III/IVe & 8396.59456 & 8 & 170 & 2.659 & 2.602 \\
128293 & 6.76 & B3Vne & 8396.60364 & 4 & 13 & 2.633 & 2.573 \\
179419 & 6.43 & B8/9Ve & 8397.93167 & 10 & 170 & 2.741 & 2.708 \\
203699 & 6.86 & B2.5IVne & 8397.93964 & 10 & 130 & 2.660 & \\
\hline
\end{tabular}

Table 2. (available electronically) The normalized spectra (Sample)

\begin{tabular}{|c|cccccccc|}
\hline \multicolumn{1}{c}{$\lambda$} & HD 90966 & HD 91188 & HD 94366 & HD 94910 & HD 124834 & HD 128293 & HD 179419 & HD 203699 \\
\hline 6448.018 & 1.0015 & 0.9992 & 0.9369 & 1.0018 & 1.0076 & 1.0357 & 1.0062 & 1.0163 \\
6450.412 & 1.0055 & 1.0100 & 1.0310 & 1.0573 & 1.0013 & 1.0150 & 1.0176 & 1.0237 \\
6452.805 & 0.9962 & 1.0210 & 1.0165 & 1.0083 & 1.0089 & 0.9146 & 1.0119 & 1.0260 \\
\hline
\end{tabular}

Table 3. Equivalent widths $(\AA)$ of spectral lines seen in our spectra. A slash separates equivalent widths measured with different criteria (see text). The H $\alpha$ line in HD 94910 (AG Car) is blended with [NII] emission

\begin{tabular}{|c|c|c|c|c|c|c|c|c|c|c|c|c|c|}
\hline & & \multicolumn{3}{|c|}{$\lambda$ He I lines } & \multicolumn{3}{|c|}{$\lambda$ OI lines } & \multicolumn{6}{|c|}{$\lambda$ Paschen lines } \\
\hline HD & $\mathrm{H} \alpha$ & 6678 & 7065 & 7281 & 7775 & 7886 & 8446 & 8502 & 8545 & 8598 & 8665 & 8750 & 8863 \\
\hline 90966 & -32.0 & 0.5 & & & -0.5 & & -3.0 & & & & & & \\
\hline 91188 & -5.0 & 0.7 & & & 1.4 & & & 1.1 & 1.8 & 2.8 & 3.8 & 3.6 & 3.7 \\
\hline 94366 & $-6.3 /-9.0$ & & & & & & & & 2.9 & 4.2 & & & \\
\hline 94910 & -69.1 & -8.0 & -18.4 & -3.2 & & & -3.9 & & & & & & \\
\hline 124834 & $<-11.9 /-12.9$ & 0.3 & & & & & -1.2 & 1.0 & 2.1 & 3.3 & 4.0 & 3.1 & \\
\hline 128293 & $-0.1 /-7.2$ & & & & & & & & & & & & \\
\hline 203699 & $<-20.2$ & 0.5 & 0.2 & & & & -1.6 & & & & & & \\
\hline 179419 & $<-1.5 /-5.3$ & & & & & & & 0.5 & 1.1 & 3.0 & 4.4 & 6.5 & 4.6 \\
\hline
\end{tabular}


Table 4. Radial velocities $\left(\mathrm{km} \mathrm{s}^{-1}\right)$ of spectral lines seen in our spectra

\begin{tabular}{|c|c|c|c|c|c|c|c|c|c|c|}
\hline & & \multicolumn{3}{|c|}{$\lambda$ He I lines } & $\lambda$ OI lines & \multicolumn{5}{|c|}{$\lambda$ Paschen lines } \\
\hline HD & $\mathrm{H} \alpha$ & 6678 & 7066 & 7281 & 8446 & 8502 & 8545 & 8598 & 8665 & 8750 \\
\hline 90966 & -22 & & & & -20 & & & & & \\
\hline 91188 & -16 & 64 & & & & 12 & 13 & 6 & 35 & 31 \\
\hline 94366 & -25 & & & & & & & & & \\
\hline 94910 & 43 & 55 & & 33 & 38 & & & & & \\
\hline 124834 & -9 & -1 & & & -4 & & 44 & 48 & 69 & 101 \\
\hline 128293 & -51 & & & & & & & & & \\
\hline 179419 & -16 & & & & & & 68 & 28 & 71 & 50 \\
\hline 203699 & 1 & 10 & & & & & & & & \\
\hline
\end{tabular}

and van Genderen et al. (1996) for a discussion of the photometry. We have observed AG Car early in the phase of a new increase in brightness after a low-brightness phase lasting $\approx 3$ years. The star was at about $V=7^{\mathrm{m}} 6$ during our observation (see Fig. 7 where its estimated position corresponds with the head of the vertical arrow). Our spectrum shows a very strong $\mathrm{H} \alpha$ emission peak (see Fig. 5) flanked by weaker emissions probably corresponding to [NII] 6548 and 6584 , which in our spectral resolution should, in fact, not be resolved. Helium emissions are also present.

The data plotted in Fig. 7 are from different sources, viz. Kilkenny et al. (1985), van Genderen et al. (1988, 1990), Leitherer et al. (1994) and data from the LongTerm Photometry of Variables project (LTPV, Sterken 1983, 1993) and published by Manfroid et al. (1991, 1994) and Sterken et al. $(1993,1995)$.

\section{5. $H D 124834$}

Shallow, slightly symmetric double $\mathrm{H} \beta-\mathrm{H} \delta$ emissions were reported by Andersen \& Nordstrom (1977). A strong double emission was observed at $\mathrm{H} \beta$ on August 9, 1978 by Jaschek \& Jaschek (1992). Our spectra show $\mathrm{H} \alpha$ and OI $\lambda 8446$ in emission. Paschen lines and He I $\lambda 6678$ are in absorption. The radial velocities of the P11-P15 Paschen absorption lines show a smooth negative progression with an average value of $66 \pm 22 \mathrm{~km} \mathrm{~s}^{-1}$ whereas the $\mathrm{H} \alpha$ and OI $\lambda 8446$ emission lines and the He $\lambda 6678$ line show velocities remarkably different with a mean of $5 \pm 3 \mathrm{~km} \mathrm{~s}^{-1}$.

\section{6. $H D 128293$}

Emission in $\mathrm{H} \alpha$ and $\mathrm{H} \gamma$ with a mean equivalent width of $3.2 \AA$ was observed by Buscombe (1970). His radial velocity data (Buscombe 1962) show strong variations of both the emission and absorption component on time scales of 2-3 months. $\mathrm{H} \beta$ double central emission with a broad absorption line on August 8, 1978 was reported by Jaschek \& Jaschek (1992). Our spectra also show the presence of weak $\mathrm{H} \alpha$ emission. This star was observed by one of us in the survey reported by Sterken \& Jerzykiewicz (1983), and was classified $\operatorname{var} * *$, i.e. significant light variations (in the Strömgren $b$ band) were detected in HD 128293, the second comparison star HD 131058, or in both. The associated $u, v$ and $y$ data have never been analysed, and could perhaps answer the question which of both stars is the dominant variable. Unfortunately, the data could not be retrieved by us from their repository.

\section{7. $H D 179419$}

Several years ago this late-type B star showed a weak diffuse roughly symmetric $\mathrm{H} \beta$ emission (Andersen \& Nordstrom 1977). Our spectrum also shows a low-emission star characterized by a weak $\mathrm{H} \alpha$ emission upon broad absorption wings. Paschen lines are observed in absorption. The discrepant values of the radial velocities found in P13 and P11 could indicate contamination by CaII and/or NI at P13 and FeI $\lambda \lambda 8864$ at P11. When comparing the mean radial velocity of Paschen lines (54 $\left.\pm 17 \mathrm{~km} \mathrm{~s}^{-1}\right)$ with the $\mathrm{H} \alpha$ radial velocity $\left(-16 \mathrm{~km} \mathrm{~s}^{-1}\right)$ it seems that the envelope is radially moving out.

\section{8. $H D 203699$}

Observations in the $21 \mathrm{~cm}$ hydrogen line have revealed that this star is immersed in a high-velocity interstellar cloud probably arising from an old supernova remnant (Little et al. 1994). The optical spectrum has shown central double emissions at $\mathrm{H} \beta, \mathrm{H} \gamma$ and $\mathrm{H} \delta$ upon underlying absorption lines on August 9, 1978 (Jaschek \& Jaschek 1992). $\mathrm{H} \alpha$ emission with $W_{\alpha}=-5.1 \AA$ was observed on October 22, 1981 by Andrillat (1983). Our spectrum shows a stronger $\mathrm{H} \alpha$ emission and weak He $\lambda 6678-7066$ absorptions. OI $\lambda 7775-8446$ are in emission. Paschen lines seem to be completely filled by emission.

Acknowledgements. This research was supported by the Dirección de Investigación de la Universidad de Concepción, Chile, PI \# 95.11.11.1-1. This research has made use of data obtained through the High Energy Astrophysics Research Center Online Service, provided by the NASA-Goddard Space Flight center. CS acknowledges a research grant from the Belgian Fund for 
Scientific Research (NFWO). Part of the data discussed in this paper were collected under observing program ESO 57D-0133. This research has made use of the SIMBAD database, operated at CDS, Strasbourg, France.

\section{References}

Albers H., 1969, ApJ 156, 37

Andersen J., Nordstrom B., 1977, A\&AS 29, 309

Andrillat Y., 1983, A\&AS 53, 319

Andrillat Y., Jaschek M., Jaschek C., 1988, A\&AS 72, 129

Balona L.A., Cuypers J., Marang F., 1992, A\&AS 92, 533

Buscombe W., 1962, MNRAS 124, 189

Buscombe W., 1970, MNRAS 148, 79

Cramer N., 1984, A\&A 141, 215

Cramer N., 1994, Applications de la photométrie de Genève aux étoiles B et à l'extinction interstellaire, Thèse 2692, Université de Genève

Cramer N., Maeder A., 1979, A\&A 78, 305

Hauck B., Mermillod M., 1990, A\&AS 86, 107

Hoffleit D., Saladyga M., Wlasuk P., 1983, A Supplement to the Bright Star Catalogue, Yale University Observatory, New Heaven, Connecticut, U.S.A.

Humphreys R.M., Davidson K., 1994, PASP 106, 1025

Jaschek C., Jaschek M., 1992, A\&AS 95, 535

Jaschek M., Jaschek C., Andrillat Y., 1993, A\&AS 97, 781

Kilkenny D., Whittet D.C.B., Davies J.K., et al., 1985, SAAO Circ. 9, 55
Leitherer C., Allen R., Altner B., Damineli A., Drissen L., et al., 1994, ApJ 428, 292

Little J.E., Dufton P.L., Keenan F.P., Conlon E.S., Davies R.D., 1994, ApJ 427, 267

Manfroid J., Sterken C., Bruch A., et al., 1991, A\&AS 87, 481

Manfroid J., Sterken C., Cunow B., et al., 1994, A\&AS 109, 329

Oudmaijer R.D., Van der Veen W.E.C.J., Waters L.B.F.M., Trams N.R., Waelkens C., Engelsman E., 1992, A\&AS 96, 625

Reed B.C., 1996, PASP 108, 61

Rufener F., 1988, Catalogue of Stars measured in the Geneva Observatory Photometric system (fourth edition), Genève (1988)

Sterken C., 1983, The Messenger 33, 10

Sterken C., 1993, in Precision Photometry, D. Kilkenny, Lastovica E., Menzies J. (eds.), South African Astronomical Observatory, p. 57

Sterken C., Jerzykiewicz M., 1983, Acta Astr. 33, 41

Sterken C., Manfroid J., Anton K., et al., 1993, A\&AS, 102, 79

Sterken C., Manfroid J., Beele D., et al., 1995, A\&AS 113, 31

van Genderen A.M., Thé P.S., Augusteijn Th., et al., 1988, A\&AS 74, 453

van Genderen A.M., Thé P.S., Heemskerk M., et al., 1990, A\&AS 82, 189

van Genderen, A.M., Sterken, C., de Groot, M.J.H., 1996, A\&A (in press) 